\title{
Stochastic Approach for Witnessing the Incubation Period of a Patient
}

\author{
Vinoth Raman $^{1}$, Kannadasan Karuppaiah ${ }^{2}$, Subash Chandrabose Gandhi ${ }^{3}$ \\ ${ }^{1}$ Quality Measurement and Evaluation Department, Deanship of Quality and Academic Accreditation, Imam Abdulrahman Bin Faisal \\ University, Dammam, Saudi Arabia \\ ${ }^{2}$ Department of Community Medicine, Melmaruvathur Adhiparasakthi Institute of Medical Sciences and Research, Melmaruvathur, India \\ ${ }^{3}$ Department of Community Medicine, Aarupadai Veedu Medical College \& Hospital, Puducherry, India
}

Email address:

vrrangan@iau.edu.sa (V. Raman), statkannadasan@gmail.com (K. Karuppaiah), subashstat@gmail.com (S. C. Gandhi)

\section{To cite this article:}

Vinoth Raman, Kannadasan Karuppaiah, Subash Chandrabose Gandhi. Stochastic Approach for Witnessing the Incubation Period of a Patient. Biomedical Statistics and Informatics. Vol. 6, No. 3, 2021, pp. 54-58. doi: 10.11648/j.bsi.20210603.13

Received: June 10, 2021; Accepted: June 24, 2021; Published: August 23, 2021

\begin{abstract}
The spread of HIV remains a huge investigation in this present environment. A Mathematical or Statistical model must be developed for estimating parameters related to the epidemic, the death rate of affected cells or the infectious viral production rate. Inability to carry out people evaluates their HIV status has led to widespread lack of correct and comprehensive data on HIV infection, while an individual first involved. Stochastic model measures the predicted point of threshold through discrete and continuous distribution attained by many researchers in last two decades. This paper develops a stochastic model for the time of HIV epidemic in a homosexual population. Expected time of incubation period derived through shock model approach. The fitting of information sets generated through simulation methods that the Alpha statistical distribution ought to be assumed because the epidemic distribution planned the time of stochastic model to search out HIV epidemics. To check the validity of analytical arguments and to explore the dynamics of disease above the epidemic threshold, this study concludes, the possible significance of the result is that transmit HIV in incubation stage is quicker as the intensity of the immune system is lower.
\end{abstract}

Keywords: Distribution, HIV/AIDS, Infection, Incubation Period and Stochastic Model

\section{Introduction}

Stochastic events start right from the stages of associate grade infection with the limits. The insignificant space of viruses running into the body will set up associate term disease, which may be a natural situation. Design state of breakthrough of AIDS is the model by Tan and $\mathrm{Wu}$ [1] that may be a random interpretation of the model by Perelson et al. [2] and Schenzle [3]. The HIV theoretical models focused on a parametric design by Tan and $\mathrm{Wu}$ [1], with few exceptions. The random organic \{biological process\} transforms throughout the habitual part through the mutation process.

As sexuality plays a significant role in individual survival, the microorganism takes the vulnerability of being transmitted from one affected individual to either. The degree of infected or non-infected human being by the pattern of their intimate behaviour. Since the behavior is stochastic, the stage for a vulnerable to become associate degree infective is unpredictable, so the dynamics of the unfold of HIV presents many perplexing difficulties in its comprehension indeed within the case of a selected population of transfusion connected processes of AIDS [4]. Incubation period of HIVtainted individual is a term from time of infection to point of first diagnosis, a strategic disease correlated with AIDS. According to Medley et al. [4], one among the putting options of no inheritable immunological disorder syndrome is that the moment to be each long and variable. The date of epidemic isn't analyzed most times. The seroconversion stage (i.e., the time associate degree infected individual become HIV positive) is classified in several cases. Latent interval between point of disease and time of seroconversion is tiny (weeks) compared to time (years) HIV.

Developed a brand alternative technique for evaluating the term of AIDS supported age distributions. HIV expressed 
point of divergence between age at time analyzing and age at time epidemic. Assumptive independence between age at break of infection and end the age distribution of diagnosed AIDS cases was applied because of the convolution product between the distributions of the infected patient's age and the incubation stages. AIDS incubation time might be calculable from the age of distribution of different HIV-involved cases and diagnosed AIDS cases [5].

Estimated the HIV virus and the period of AIDS by victimization information from 363 sero-prevalent (i.e. individuals who were AIDS free at admission) Korean AIDS victims (comprising fifty-nine sero-incident cases). The Authors, prepared 2 designs for ascribing the strange times since seroconversion were, fitting Weibull regression with the indicator of matured CD4+T cell count for sero-incident cohorts and using a variable effects model with $\mathrm{CD} 4+\mathrm{T}$ cell count as a return for perennial measures from the days since seroconversion will be extracted [6]. Pereson et al. [7] had considered the established model and Tan and Xiang [8] had a state model, contracting with a random model growth of HIV population that moves over the doctrine of the antibiotic of HIV and so the life-cycle HIV, then permits the assembly of non-infectious (defective) free HIV to scale back the severity of HIV in a very HIV-infected individual undergoing a treatment. The infection time in incubation stage and fieldsegregation on the sensitive is developed [15].

\section{Reporting of Stochastic Period}

To contemplate a skewed population whose tonality of transmission is through heterosexual activity. Assume that at time $t=0$, a brand new representative of tested HIV negative enters the state and approaches a member of inclined. Let the sexual contacts occur at random point assumed to follow the Alpha Poisson distribution Pillai [9] and Anil [10] with parameters ' $a$ ' and ' $\alpha$ ' given as

$$
\begin{gathered}
\mathrm{P}_{\mathrm{a}, \alpha}(\mathrm{n}, \mathrm{t})=\sum_{0}^{\infty}(-1)^{\mathrm{k}}\left(\begin{array}{c}
\mathrm{k}+\mathrm{n} \\
\mathrm{k}
\end{array}\right) \frac{(\mathrm{at})^{\alpha(\mathrm{k}+\mathrm{n})}}{\Gamma(\alpha(\mathrm{k}+\mathrm{n})+1)}, \mathrm{a}>0,0<\alpha<1, \mathrm{n}=0,1,2 \ldots \\
=\frac{\mathrm{e}^{-(\mathrm{at})^{\alpha}\left(\mathrm{at}^{\alpha}\right)^{\mathrm{k}}}}{\Gamma(\alpha \mathrm{k}+1)}, \mathrm{a}>0,0<\alpha<1
\end{gathered}
$$

$G(t)$ the distribution function of the inter-arrival between the contacts, which follows Mittag-Leffler distribution. The function of Mittag-Leffler distribution is given by Pillai and Anil [9, 10],

$$
\mathrm{G}_{\mathrm{a}, \alpha}(\mathrm{t})=\sum_{0}^{\infty} \frac{(-1)^{\mathrm{k}}(\mathrm{at})^{\alpha}}{\Gamma(\alpha \mathrm{k}+1)}, \mathrm{t} \geq 0, \mathrm{a}>0,0<\alpha \leq 1
$$

Mittag-Leffler distributions can be applied as expectedtime distributions, yet as first-passage time distributions sure renewal processes with geometric exponential as waitingtime distribution. They'll even be used in dependableness modeling as another for exponential period distribution. The normal and generalized Mittag-Leffler functions interpolate between an exponential law and Stevens' law behavior of phenomena ruled by normal kinetic equations and their counterparts [11].

Let the incubation period of the HIV individual diagrammatical by the variant $\mathrm{T}$, we have an incubation period HIV by a random model supported the later assumptions. Sexual contact is the sources of HIV transmission. Damages to people square measure caused by transmission HIV at every contact and the inter-arrival between the contacts are freelance distributed random variables. Damage acting on the immune of an infected individual is non-linear and additive. The overall harm caused exceeds a strength Y itself may be a variant. Methods generates the contacts; the sequence of damages and threshold are independent.

Let $X_{i}$ be the incubation period arising due to HIV

$$
\begin{gathered}
\qquad(t)=P\{\text { no infection }(0, t]\}=P\{T>t\} \\
=\sum_{0}^{\infty} \mathrm{P}[\text { exactly k contact in }(0, \mathrm{t}] \text { with intensity }] \times \mathrm{P} \text { [exactly k contact in }(0, \mathrm{t}] \text { with intensity } \alpha \text { ] }
\end{gathered}
$$

transmission during the $i^{\text {th }}$ Contact and $X_{i}$ are i. i. d for $i=1,2 \ldots k$

$\mathrm{g}()=$. The c. d. f of $\mathrm{X}$ by taking $X_{i}=X$ for $i=1,2 \ldots k$

$\mathrm{V}_{\mathrm{k}}(\mathrm{t})=$ The probability of exactly $\mathrm{k}$ contacts in $(0, \mathrm{t}]$

$\mathrm{g}^{*}(\mathrm{~s})=$ Laplace transform of $g($.

$\mathrm{g}_{\mathrm{k}}()=$. The probability density function of random variable $\sum_{0}^{\infty} \mathrm{X}_{\mathrm{i}}$, which is the $k^{\text {th }}$ convolution of $g($.

$Y=$ Random variable denoting the incubation period threshold, which follows exponential distribution with parameter $\mu$

$f()=$. The probability density function of antigenic diversity threshold of $Y$, has Alpha Poisson distribution with parameters ' $\mu$ 'so that

$$
P[Y<y]=\left[2 e^{-\mu x}-e^{-2 \mu x}\right], y>0, \mu>0
$$

$\mathrm{P}[\mathrm{X}<\mathrm{Y}]=$ The probability that damaged caused in a single contact is less than the threshold $Y$.

$S(t)=\mathrm{P}$ [no infection in $(0, \mathrm{t}]]=P[T>t]$ from the above assumptions with nonlinear damage process acting on the immune system, we have the following Theorem 1.

\section{Theorem 1}

If the estimates of contacts are an Alpha Poisson process with parameters ' $a$ ' and ' $\alpha$ ' and inter-contact time is a MittagLeffler distribution while the threshold level is an Exponentiated exponential distribution with parameter ' $\mu$ ', thus the probability density function of seroconversion time is a three parameter Weibull distribution

Proof: 


$$
=\sum_{0}^{\infty} \mathrm{V}_{\mathrm{k}}(\mathrm{t}) \mathrm{P}\left\{\sum_{0}^{\infty} \mathrm{X}_{\mathrm{i}}<\mathrm{Y}\right\}
$$

Where $V_{k}(t)=$ probability of exactly $\mathrm{k}$ contacts in $(0, \mathrm{t}]$ with intensity ' $\alpha$ ' which is the Alpha Poisson distribution with parameter 'a' and ' $\alpha$ '

$$
\begin{gathered}
=\sum_{0}^{\infty} \frac{e^{-(a t)^{\alpha}}\left(a t^{\alpha}\right)^{k}}{\Gamma(\alpha k+1)}, a>0,0<\alpha \leq 1 \\
P\left\{X_{1}+X_{2}+\ldots \ldots X_{k}<\left(Y_{1}, Y_{2}\right)\right\}=\int_{0}^{\infty} g_{k}(X)\left[2 e^{-\mu x}-e^{-2 \mu x}\right] \\
P\left\{\sum_{i=1}^{\infty} X_{i}<Y\right\}=\left[2 g^{*}(\mu)\right]^{k}-\left[g^{*}(2 \mu)\right]^{k}
\end{gathered}
$$

where $g_{k}(X)=$ P.d. f of $\sum_{i=1}^{\infty} X_{i}$ and $g^{*}(\mu)=$ Laplace is the Transformation of $g(x)$

$$
\begin{aligned}
& S(t)=\sum_{0}^{\infty} V_{k}(t)\left[2 g^{*}(\mu)\right]^{k}-\left[g^{*}(2 \mu)\right]^{k}=\sum_{0}^{\infty} \frac{e^{-(a t)^{\alpha}}\left(a t^{\alpha}\right)^{k}}{\alpha k+1}\left[2 g^{*}(\mu)\right]^{k}-\left[g^{*}(2 \mu)\right]^{k} \\
& =e^{-(a t)^{\alpha}} \sum_{0}^{\infty} \frac{\left[\left(a t^{\alpha}\right)^{k} g^{*}\left(2 g^{*}(\mu)\right)\right]^{k}}{\alpha k+1}-e^{-(a t)^{\alpha}} \sum_{0}^{\infty} \frac{\left[\left(a t^{\alpha}\right)^{k} g^{*}\left(g^{*}(2 \mu)\right)\right]^{k}}{\alpha k+1}=e^{-(a t)^{\alpha}}\left[1-\left(2 g^{*}(\mu)\right)\right]-e^{-(a t)^{\alpha}}\left[1-\left(g^{*}(2 \mu)\right)\right] \\
& L(t)=1-S(t)=1-e^{-\left\{a^{\alpha}\left[1-g^{*}\left(2 g^{*}(\mu)\right)\right] t^{\alpha}\right\}}-e^{-\left\{a^{\alpha}\left[1-g^{*}\left(g^{*}(2 \mu)\right)\right] t^{\alpha}\right\}}
\end{aligned}
$$

Since the probability density function $\mathrm{X}_{\mathrm{i}}$ follows Mittag-Leffler, then

$$
g^{*}(\mu)=\frac{a^{\alpha}}{a^{\alpha}+\mu^{\alpha}} \Rightarrow 1-g^{*}(\mu)=\frac{\mu^{\alpha}}{a^{\alpha}+\mu^{\alpha}}
$$

and

$$
g^{*}(2 \mu)=\frac{a^{\alpha}}{a^{\alpha}+2 \mu^{\alpha}} \Rightarrow 1-g^{*}(\mu)=\frac{2 \mu^{\alpha}}{a^{\alpha}+2 \mu^{\alpha}}
$$

Thus

$$
L(t)=1-e^{-\left\{\frac{2 a^{\alpha} \mu^{\alpha}}{a^{\alpha}+\mu^{\alpha}}\right\}}-e^{-\left\{\frac{2 a^{\alpha} \mu^{\alpha}}{a^{\alpha}+2 \mu^{\alpha}}\right\}}
$$

The probability density function of seroconversion time $\mathrm{T}$ is

$$
\begin{gathered}
\Psi(t)=\left\{\frac{2 a^{\alpha} \mu^{\alpha} \alpha}{a^{\alpha}+\mu^{\alpha}}\right\} t^{\alpha-1} e^{-\left\{\frac{2 \alpha^{\alpha} \mu^{\alpha}}{a^{\alpha}+2 \mu^{\alpha}} t^{\alpha}\right\}}-\left\{\frac{2 a^{\alpha} \mu^{\alpha} \alpha}{a^{\alpha}+2 \mu^{\alpha}}\right\} t^{\alpha-1} e^{-\left\{\frac{2 \alpha^{\alpha} \mu^{\alpha}}{a^{\alpha}+2 \mu^{\alpha}} t^{\alpha}\right\}} \\
t \geq 0, a>0>, 0<\alpha \leq 0 \text { and } \mu>0
\end{gathered}
$$

This is the form of three parameter Weibull distribution.

\section{Probability of Incubation Period}

The probability of incubation period is calculated for the various intervals by defining

$$
\begin{gathered}
p_{i}=\int_{t_{i}}^{t_{i+1}} \Psi(t) d t \text { for } i=1,2,3 \ldots \\
p_{i}=\int_{t_{i}}^{t_{i+1}} \frac{2 a^{\alpha} \mu^{\alpha} \alpha}{a^{\alpha}+\mu^{\alpha}} t^{\alpha-1} e^{-\left\{\frac{2 a^{\alpha} \mu^{\alpha} \alpha}{a^{\alpha}+\mu^{\alpha}}\right\}} d t-\int_{t_{i}}^{t_{i+1}} \frac{2 a^{\alpha} \mu^{\alpha} \alpha}{a^{\alpha}+\mu^{\alpha}} t^{\alpha-1} e^{-\left\{\frac{2 a^{\alpha} \mu^{\alpha} \alpha}{\left.a^{\alpha}+2 \mu^{\alpha}\right\}}\right.} \text { for } i=1,2,3 \ldots \\
t \geq 0, a>0>, 0<\alpha \leq 0 \text { and } \mu_{1}>0, \mu_{2}>0
\end{gathered}
$$




$$
\begin{gathered}
p_{i}=\frac{2 a^{\alpha} \mu^{\alpha} \alpha}{a^{\alpha}+\mu^{\alpha}} \int_{t_{i}}^{t_{i+1}} t^{\alpha-1} e^{-\left\{\frac{2 a^{\alpha} \mu^{\alpha}}{a^{\alpha}+\mu^{\alpha}} t^{\alpha}\right\}} d t-\frac{2 a^{\alpha} \mu^{\alpha} \alpha}{a^{\alpha}+\mu^{\alpha}} \int_{t_{i}}^{t_{i+1}} t^{\alpha-1} e^{-\left\{\frac{2 a^{\alpha} \mu^{\alpha}}{a^{\alpha}+2 \mu^{\alpha}} t^{\alpha}\right\}} d t \text { for } i=1,2,3 \ldots \\
t \geq 0, a>0>, 0<\alpha \leq 0 \text { and } \mu_{1}>0, \mu_{2}>0 \\
\text { Let } z=\frac{2 a^{\alpha} \mu^{\alpha} \alpha}{a^{\alpha}+\mu^{\alpha}} t^{\alpha} \Rightarrow \alpha t^{\alpha-1} d t=\frac{d z}{\frac{2 a^{\alpha} \mu^{\alpha} \alpha}{a^{\alpha}+\mu^{\alpha}}}=\frac{d z}{c}, \text { where } c=\frac{2 a^{\alpha} \mu^{\alpha} \alpha}{a^{\alpha}+\mu^{\alpha}}
\end{gathered}
$$

\section{Measuring Incubation Period}

The expected time to incubation period is

$$
\begin{gathered}
E(T)=\int_{0}^{\infty} \Psi(t) d t=\int_{0}^{\infty} t \frac{2 a^{\alpha} \mu^{\alpha} \alpha}{a^{\alpha}+\mu^{\alpha}} t^{\alpha-1} e^{-\left\{\frac{2 a^{\alpha} \mu^{\alpha} \alpha}{a^{\alpha}+\mu^{\alpha}} t^{\alpha}\right\}} d t-\int_{0}^{\infty} t \frac{2 a^{\alpha} \mu^{\alpha} \alpha}{a^{\alpha}+2 \mu^{\alpha}} t^{\alpha-1} e^{-\left\{\frac{2 a^{\alpha} \mu^{\alpha} \alpha}{a^{\alpha}+2 \mu^{\alpha}} t^{\alpha}\right\}} d t \\
=\frac{2 a^{\alpha} \mu^{\alpha} \alpha}{a^{\alpha}+\mu^{\alpha}} \int_{0}^{\infty} t t^{\alpha-1} e^{-\left\{\frac{2 a^{\alpha} \mu^{\alpha} \alpha}{a^{\alpha}+\mu^{\alpha}} t^{\alpha}\right\}} d t-\frac{2 a^{\alpha} \mu^{\alpha} \alpha}{a^{\alpha}+2 \mu^{\alpha}} \int_{0}^{\infty} t t^{\alpha-1} e^{-\left\{\frac{2 a^{\alpha} \mu^{\alpha} \alpha}{a^{\alpha}+2 \mu^{\alpha}} \alpha\right\}} d t \\
\text { Let } c_{1}=\frac{2 a^{\alpha} \mu^{\alpha}}{a^{\alpha}+\mu^{\alpha}} \text { and } y_{1}=\left(\frac{2 a^{\alpha} \mu^{\alpha}}{a^{\alpha}+\mu^{\alpha}}\right) t^{\alpha} ; \text { Let } c_{2}=\frac{2 a^{\alpha} \mu^{\alpha}}{a^{\alpha}+2 \mu^{\alpha}} \text { and } y_{2}=\left(\frac{2 a^{\alpha} \mu^{\alpha}}{a^{\alpha}+2 \mu^{\alpha}}\right) t^{\alpha}
\end{gathered}
$$

then; $t^{\alpha}=\frac{y_{1}}{c_{1}} \Rightarrow \alpha t^{\alpha-1} d t=\frac{d y_{1}}{c_{1}}$ and $\alpha t^{\alpha} d t=\left(\frac{y_{1}}{c_{1}}\right)^{\frac{1}{\alpha}} \frac{d y_{1}}{c_{1}}$ and $t^{\alpha}=\frac{y_{2}}{c_{2}} \Rightarrow \alpha t^{\alpha-1} d t=\frac{d y_{2}}{c_{2}}$ any $\alpha t^{\alpha} d t=\left(\frac{y_{2}}{c_{2}}\right)^{\frac{1}{\alpha}} \frac{d y_{2}}{c_{2}}$

$$
\begin{gathered}
\therefore E(T)=\int_{0}^{\infty} e^{-y_{1}}\left(\frac{y_{1}}{c_{1}}\right)^{1 / \alpha} \frac{d y_{1}}{c_{1}}-\int_{0}^{\infty} e^{-y_{2}}\left(\frac{y_{2}}{c_{2}}\right)^{1 / \alpha} \frac{d y_{2}}{c_{2}} \\
=\frac{1}{c_{1}{ }^{1 / \alpha}} \int_{0}^{\infty} e^{-y_{1}} y_{1}{ }^{1 / \alpha} d y_{1}-\frac{1}{c_{2}{ }^{1 / \alpha}} \int_{0}^{\infty} e^{-y_{2}} y_{2}{ }^{1 / \alpha} d y_{2}=\left\{\frac{1}{c_{1}{ }^{1 / \alpha}}-\frac{1}{c_{2}{ }^{1 / \alpha}}\right\} \Gamma\left(\frac{1}{\alpha}+1\right) \\
E(T)=\left\{2\left(\frac{a^{\alpha}+\mu^{\alpha}}{a^{\alpha} \mu^{\alpha}}\right)^{\frac{1}{\alpha}}-\left(\frac{a^{\alpha}+2 \mu^{\alpha}}{2 a^{\alpha} \mu^{\alpha}}\right)^{\frac{1}{\alpha}}\right\} \Gamma\left(\frac{1}{\alpha}+1\right)
\end{gathered}
$$

\section{Numerical Illustration}

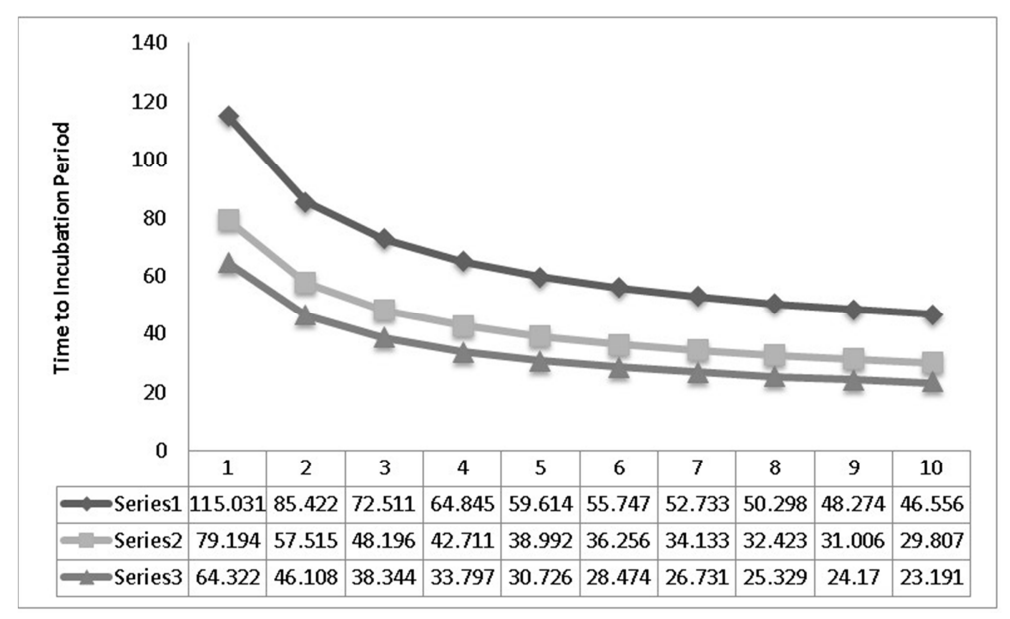

Figure 1. Survival of the Patient. 


\section{Discussion and Conclusion}

From figure 1 we noticed that for defined ' $\alpha$ ', $\mu$ and ' $a$ ' (constant rate) increases the mean of incubation period decreases. Likewise, if ' $a$ ' is fixed and $\mu$ (infection threshold) may increase, the same Expected time to incubation period is identified i.e., decreasing. The overall intensity of the incubation period of the affected partner increases as the meantime of incubation period decreases. The possible significance of the result is that the transmission of HIV in incubation period is quicker as the intensity of the immune system is lower.

Stochastic model determines expected time of threshold through various discrete and continuous distribution developed by analysts in last two decades. For further knowledge concerning to the stochastic model, one can refer to Subramanian et al. [12], Pradeep Sukla et al. [13] and Thirumurugan and Vinoth [14].

\section{References}

[1] Tan, W. Y and Wu, H. (1998). Stochastic modeling of the dynamics of CD4+ T-cell infection by HIV and some Monte Carlo studies. Math. Biosci. Vol: 147, pp. 173-205.

[2] Perelson, A. S, Kirschner, D. E and de Boer, R. (1993). Dynamics of HIV infection of CD4+ T cells. Math. Biosci. Vol: 114 , pp. 81-125.

[3] Schenzle, D. (1994). A model for AIDS pathogenesis. Stat. Med. Vol: 13, pp. 2067-2079.

[4] Medley, G. F., Billard, L., Cox, D. R. and Anderson, R. M. (1988). The distribution of the incubation period for the acquired immunodeficiency syndrome (AIDS). Proceeding of the Royal Society of London, Series B, Biological Sciences, Vol. 233, No. 1272, pp. 367-377.

[5] Chevret. S., Costagliola, D., Lefrere, and J. J and Valler on, A. J. (1992). A new approach to estimating AIDS incubation times: results in homosexual infected men. Journal of Epidemiology Community Health, Vol. 46 (6), pp. 582-586.
[6] Lee, S. (1999). Estimation of the maturity of HIV and the incubation period of AIDS patients. http://www.tilastokeskus.fi/isi99/proceedings/arkisto/varasto/l ee_0375.pdf.

[7] Pereson, A. S., Neumann, A. U., Markowitz, M., Leonard, J. $\mathrm{M}$ and Ho, D. D. (1996). HIV-1 dynamics in vivo viron clearence rate, infected cell life span, and viral generation time. Science New Science New Series, Vol. 271, pp. 1582-1586.

[8] Tan and Xiang, Z. (1999). Stochastic Modelling of the dynamics of HIV Pathogenesis under treatment by anti-viral drugs in HIV-infected individuals. Mathematical bioscience, Vol. 156, pp. 69-94.

[9] Pillai, R. N. (1990). On Mittag-Leffler function and related distributions, Annals of the Institute of statistical Mathematics, Vol: 42, pp. 157-161.

[10] Anil. V. (2001). A generalized distribution and its application. Journal of the Kerala statistical Association, Vol 12: pp. 11-20.

[11] Mathai, A. M., Saxena, R. K., Haubold, H. J. (2006). A certain class of Laplace transforms with applications to reaction and reaction-diffusion equations. Atrophy's. Space Sci. Vol: 305, pp. 283-288.

[12] Subramanian, C, R. Rajivgandhi and R. Vinoth. (2012), Estimation of the Generalized Logistic Distribution based on the Expected time in Shock Model, Global Journal of Mathematical Sciences: Theory and Practical, Vol. 4, No. 12, pp. 57-62.

[13] Pradeep Sukla, D. Raja, D. Jegadeesh Ramasamy and R. Vinoth. (2013), Preventing Threshold in Human Immune Virus of Infected Persons through Statistical Model, International Journal of Pharmaceutical Science and Health Care, Vol. 1 (2), pp. 43-46.

[14] Thirumurugan, A and R. Vinoth. (2016), "Time to Survival of HIV Environment of the Infected Patients", Journal of Reliability and Statistical Studies, Vol. 9 (2), pp. 91-98.

[15] Shangguan, D., Liu, Z., Wang, L., Tan R. (2021). A stochastic epidemic model with infectivity in incubation period and homestead-isolation on the susceptible. Journal of Applied Mathematics and Computing. https://doi.org/10.1007/s12190021-01504-1. 